\title{
A Method for Simulating Wind Conditions During Atmospheric Stagnation Periods
}

\author{
Edwin L. MEYer, JR. \\ Civil Works Directorate, Office of the Chief of Engineers, Washington, D. C. \\ AND JIMMIE E. QUON \\ Dept. of Civil Engineering, Northwestern University, Evanston, Ill. \\ (Manuscript received 30 November 1971, in revised form 21 March 1972)
}

\begin{abstract}
A method is presented whereby meteorological conditions encountered during air pollution episodes may be simulated. Meteorological conditions are expressed in terms of atmospheric stability, mixing depth, surface temperature, present wind direction, present wind speed, and wind direction and speed variability. Realistic conditions are obtained by varying stability, mixing depth and surface temperature diurnally in accordance with patterns observed during past episodes. Wind direction and speed are determined by using a digital computer to generate values from empirical frequency distributions and random number generator routines. Once an initial wind direction and speed are selected, subsequent directions are obtained by generating wind direction changes from wind direction variability frequency distributions and adding these changes to former values. Wind speeds are generated from conditional frequency distributions appropriate for each wind direction. Comparison of computer generated and observed wind directions and speeds failed to reveal any significant statistical differences.

Generating wind conditions stochastically from empirical frequency distributions provides one with greater flexibility in testing the effectiveness of hypothetical air pollution control strategies during emergencies. Repeated simulations of wind conditions in this manner, when used as meteorological input to a dispersion model, may provide aid in evaluating different control strategies.
\end{abstract}

\section{Introduction}

There are three approaches which one may take in providing input for numerical calculations of diffusion models. The first of these is the direct use of field observations made during previous periods for the input variables of interest. The second method involves a stochastic approach, in which input variables are generated from frequency distributions derived from past observations. The third approach is that of developing functional relationships in which the variables of interest are dependent variables and all independent variables are known or specified. Upon examining the problem of providing meteorological input for use in atmospheric dispersion models, it becomes apparent that the third approach must be eliminated as there are presently no mathematical formulas relating essential meteorological variables to each other, to their values during previous time intervals, or to the time of day.

The first approach of using directly the values of meteorological variables observed during previous periods of interest is the most straightforward one, but it forces the investigator to rely exclusively on data which have already been observed. Consequently, one is unable to make observations under meteorological conditions which could conceivably happen, but which were not observed during the period studied.
The stochastic approach provides one with more flexibility in making predictions about pollutant dispersion than is possible by specifying meteorological observations directly. This technique involves constructing frequency distributions for each variable and/or dependent frequency distribution, and denoting statistical interdependencies among variables in order to obtain a realistic set of meteorological conditions.

\section{Procedure}

In these studies, meteorological conditions encountered during past air pollution episodes are simulated to provide a measure of atmospheric dilutive capacity for use in a dispersion model. Atmospheric dilutive capacity is characterized by eight variables, and may be expressed as an eight-dimensional vector:

where

$$
\mathrm{M}=\left(n_{1}, n_{2}, n_{3}, n_{4}, n_{5}, n_{6}, n_{7}, n_{8}\right),
$$

\footnotetext{
$n_{1}$ time of day

$n_{2}$ atmospheric stability conditions

$n_{3}$ atmospheric mixing depth

$n_{4}$ surface temperature

$n_{5}$ wind direction

$n_{6}$ wind speed

$n_{7}$ wind direction during the next time interval

$n_{8}$ wind speed during the next time interval
} 
Each of the elements of this vector may be dependent upon one another to varying degrees. Consequently, there are various combinations which are more likely to occur than others. For example, for a time interval $\Delta t$ of $1 \mathrm{hr}$, an $n_{5}$ representing a south wind is far more likely to be accompanied by an $n_{\tau}$ indicative of south, southeast or southwest winds than one indicating north, northeast or northwest winds. This is so because wind direction is not likely to change radically within a 1 -hr time interval. When $n_{1}$ indicates it is afternoon, one is more likely to find $n_{2}$ indicating atmospheric instability than stability; the reverse would be true during nighttime. Similarly, associated with atmospheric instability would be a relatively large mixing depth $\left(n_{3}\right)$, a relatively high wind speed $\left(n_{6}\right)$ and a relatively high temperature $\left(n_{4}\right)$, as compared to the daily average values of these elements. In addition, the relationships among elements of $\mathbf{M}$ are altered when the size of $\Delta t$ is changed. For example, when $\Delta t$ is changed from 1 to $5 \mathrm{hr}$, a combination of an $n_{5}$ south wind and an $n_{7}$ north wind becomes more likely.

Atmospheric stagnation periods have been characterized by Miller and Niemeyer (1963) as having:

1) surface wind speeds $<4 \mathrm{~m} \mathrm{sec}^{-1}$

2) wind speeds $<12.5 \mathrm{~m} \mathrm{sec}^{-1}$ at all altitudes below $6000 \mathrm{~m}$

3) a subsidence inversion below an altitude of $4700 \mathrm{~m}$

4) a persistence of the above conditions for at least $36 \mathrm{hr}$

The first step in simulating an atmospheric stagnation period is to simplify the vector in Eq. (1) by combining some of its elements. The model assumes that there is a typical diurnal pattern of atmospheric stability $\left(n_{2}\right)$, mixing depth $\left(n_{3}\right)$ and surface temperature $\left(n_{4}\right)$ during stagnation periods. These assumptions are based in part on observations by Gifford (1952), Munn and Richards (1963) and Izumi (1964), and in part upon analysis of temperature profile data obtained at Green Bay, Wisc., Peoria, Ill., and Flint, Mich., during three past stagnation periods (28 November-2 December 1962 ; 24-27 June 1963; 12-16 October 1964). Consequently, atmospheric stability, mixing depth and temperature are combined with time of day $T$ in the model, and Eq. (1) becomes

$$
\mathbf{M}=\left(T, n_{5}, n_{6}, n_{7}, n_{8}\right) .
$$

In simulating temperature and mixing depth, one specifies the average surface temperature and the peak and minimum mixing depth values, and the diurnal pattern is simulated implicitly. The peak and minimum values of mixing depth and average daily surface temperature are, of course, functions of location and time of year. In these studies, maximum daily mixing depths and average daily surface temperatures prevailing during the 28 November-2 December 1962 episode in the Chicago area are specified. During the nighttime hours (1800-0500) the minimum mixing depth is used. This simplifying assumption is made on the welldocumented urban heat island effect (Duckworth and Sandberg, 1954; DeMarrais, 1961; Pooler, 1966; Bornstein, 1968). The maximum mixing depth is varied incrementally during the daytime hours between the daily maximum value and the nightly minimum value. Surface temperature is varied hourly in accordance with an empirical function based on diurnal surface temperature patterns observed during the days of the three stagnation periods mentioned above. Hourly surface temperature values agreed closely with those observed during the 28 November-2 December 1962 episode. Day and nighttime stability conditions are specified. During the nighttime and early morning hours, adiabatic conditions are assumed to prevail beneath the mixing depth as the result of the heat island effect. The nighttime mixing depth is marked by the base of a stahle layer of air. During the late morning and afternoon hours, a superadiabatic lapse rate, characteristic of clear days, is assumed beneath the maximum mixing depth. It would have been interesting to generate daily maximum mixing depths and daily average surface temperatures stochastically. Unfortunately, the number of observations were insufficient to serve as a means for validating such a procedure.

Eq. (2) becomes more meaningful by combining elements $n_{7}$ and $n_{5}$ and elements $n_{8}$ and $n_{6}$ into wind direction change and wind speed change variables, respectively, as shown in Eqs. (3) and (4):

$$
\begin{aligned}
\Delta \theta & =n_{7}-n_{5}, \\
\Delta U & =n_{8}-n_{6} .
\end{aligned}
$$

By expressing wind direction during the present time interval as $\theta$ and wind speed as $U$, Eq. (2) becomes

$$
\mathbf{M}=(T, \theta, U, \Delta \theta, \Delta U) .
$$

The problem of simulating meteorological conditions encountered during stagnation periods has now been reduced to one of obtaining realistic combinations of the five elements of $\mathbf{M}$ in Eq. (5). Such combinations have been obtained by constructing frequency distributions for each of the latter four elements of $\mathbf{M}$ and weighting these so as to reflect the dependence of the four elements on one another and on time of day. Values are then generated stochastically from these frequency distributions using random number generating routines. Data observed at Chicago's Midway and O'Hare airports during the three stagnation periods previously noted were used in constructing the appropriate frequency distributions. Specifically, these distributions include:

1) A wind direction frequency distribution $\left(F_{\theta}\right)$ for the eight principal compass directions. 


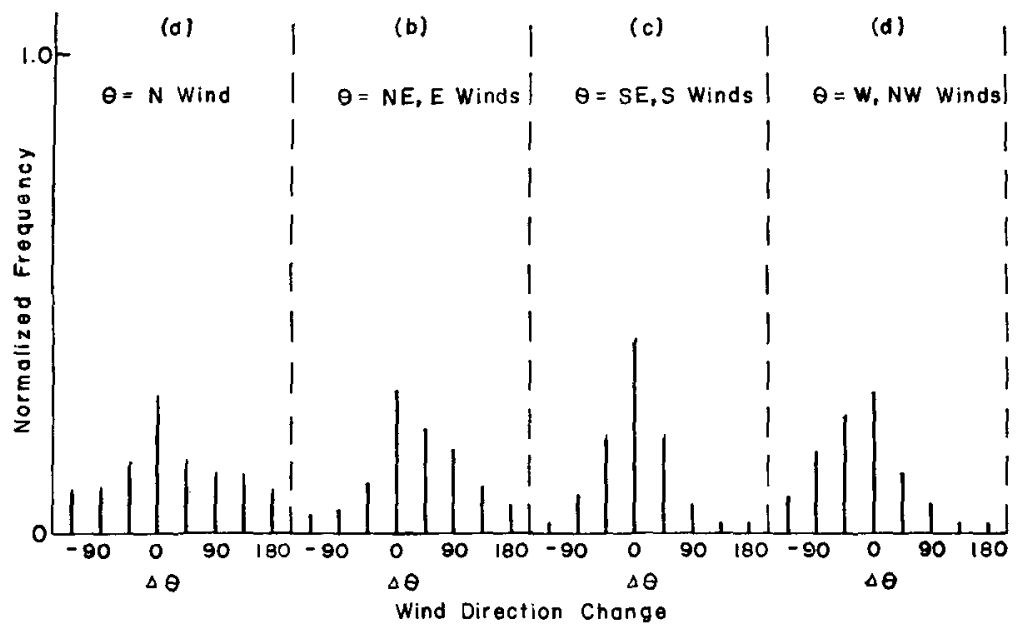

FIG. 1. Structure of a composite wind direction change frequency distribution.

2) Wind direction change $\left(F_{\Delta}\right)$ frequency distributions for 1, 2, 3 and $4 \mathrm{hr}$ intervals of time. Each of these $F_{\Delta}$ is actually a composite of four conditional $F_{\Delta}$ distributions which are dependent on the present wind direction. In this model a distinct $F_{\Delta}$ distribution was used for: (i) north winds, (ii) northeast or east winds, (iii) southeast, south or southwest winds, and (iv) west and northwest winds. Ideally, it would have been preferable to construct an $F_{\Delta}$ distribution for each of the eight principal wind directions, but the data were insufficient for this. Fig. 1 is a schematic picture of the manner in which each of the four conditional $F_{\Delta}$ distributions are weighted to generate a series of $\Delta \theta$ values similar to those observed during the three stagnation periods examined.

3) Eight conditional wind speed frequency disbributions $\left(F_{U}\right)$, one for each of the eight principal wind directions.

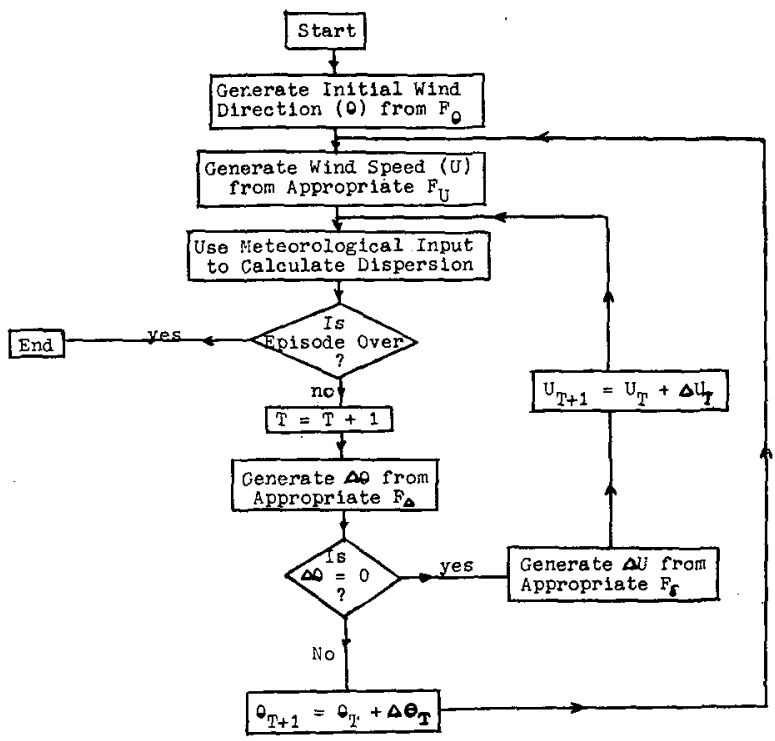

FIG. 2. Flow diagram for input of wind conditions.
4) A wind speed change frequency distribution $\left(F_{\delta}\right)$, applicable when there is no accompanying wind direction change between successive time intervals.

The wind direction, wind direction change, wind speed and wind speed change frequency distributions are utilized in the following manner: For the initial time interval in the simulated episode, a wind direction is generated from $F_{\theta}$. Next, a wind speed for this initial time interval is generated from the appropriate $F_{U}$ by using a random number generator. For the second time interval, a $\Delta \theta$ value is generated from the $F_{\Delta}$ appropriate for the $\theta$ during the first time interval, and Eq. (6) is used to compute the new wind direction

$$
\theta_{2}=\theta_{1}+\Delta \theta_{1} \text {. }
$$

A new wind speed $U_{2}$ is now generated from the conditional $F_{U}$ for $\theta_{2}$. If $\Delta \theta_{1}$ in (6) is zero, a $\Delta U$ value is generated from $F_{\delta}$ and added to $U_{1}$ to obtain $U_{2}$, i.e.,

$$
U_{2}=U_{1}+\Delta U_{1} \text {. }
$$

Values of $\theta, \Delta \theta, U$ and $\Delta U$ are obtained for subsequent time intervals in a manner identical to that described for the second time interval. Fig. 2 is a flow diagram summarizing the manner in which wind speed and direction are determined for each time interval.

Dependence of $U$ and $\Delta U$ on time of day were observed from the data. Daytime wind speeds and changes in wind speed between time intervals were approximately twice those observed at night. Consequently, generated values of $U$ and $\Delta U$ are multiplied by appropriate specified constants to exhibit diurnal wind speed patterns. No discernible diurnal wind direction pattern was apparent from the data.

\section{Results}

Six hundred and seventy-two values of $\theta$ and $\Delta \theta$ generated by random number generating routines using 


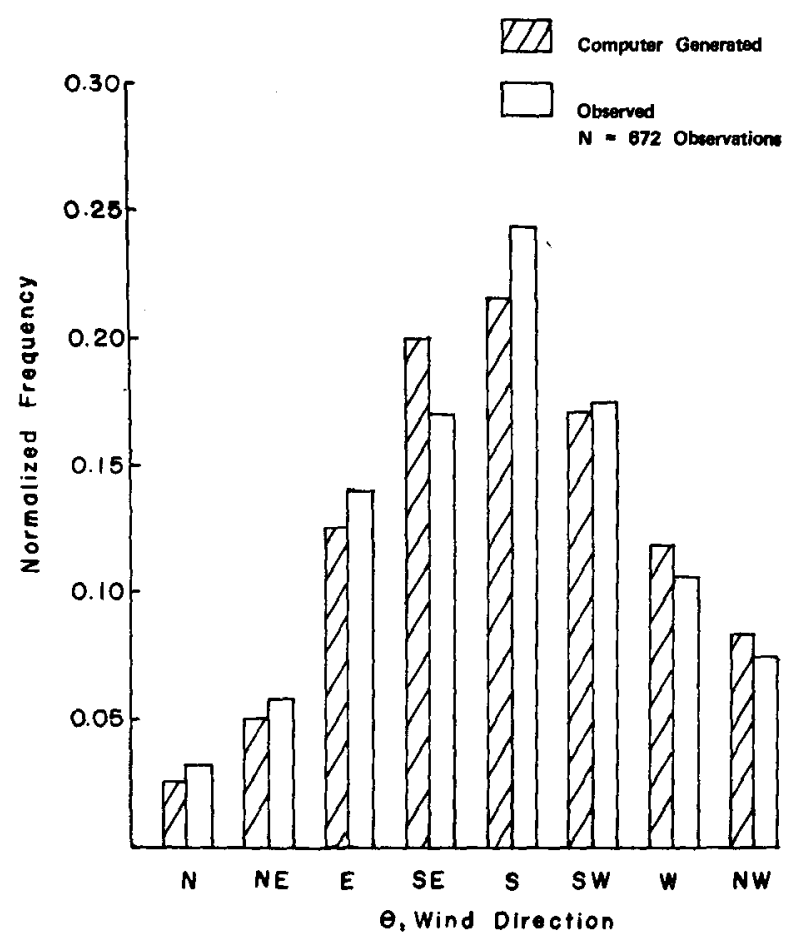

FIG. 3. Comparison of observed and computer-generated wind direction frequency distributions.

empirical frequency distributions were compared with a similar number observed during the three past episodes by chi-square analysis. Figs. 3 and 4 show the comparison between observed and generated values of $\theta$ and $\Delta \theta$, respectively. For purposes of this analysis a null hypothesis, stating that there is no real difference between observed and generated values, was formulated. A criterion of $p \leqslant 0.10$ for obtaining a chi-square value larger than the observed one was chosen as the basis for rejecting the null hypothesis. Results of chi-

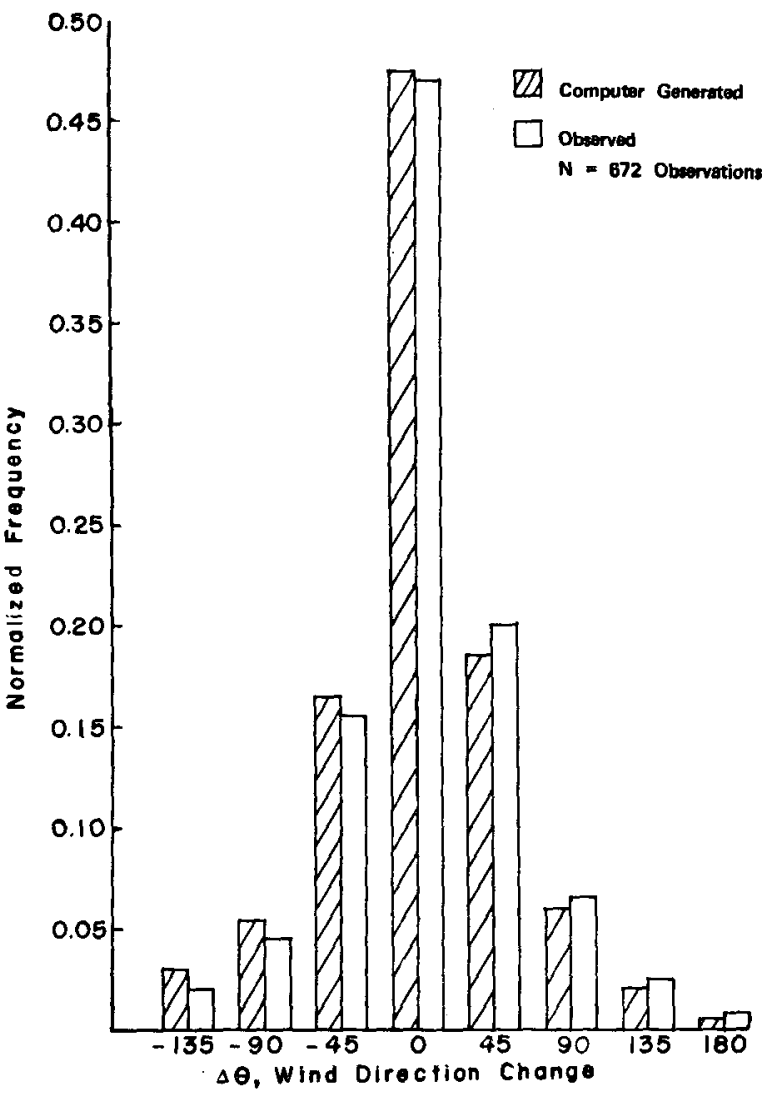

Fig. 4. Comparison of observed and computer-generated wind direction change frequency distributions.

square analysis for $\theta$ and $\Delta \theta$ are shown in Table 1 . As Table 1 indicates, the hypothesis that there is no real difference between observed and computer-generated values of $\theta$ and $\Delta \theta$ cannot be rejected with a confidence level of $90 \%$.

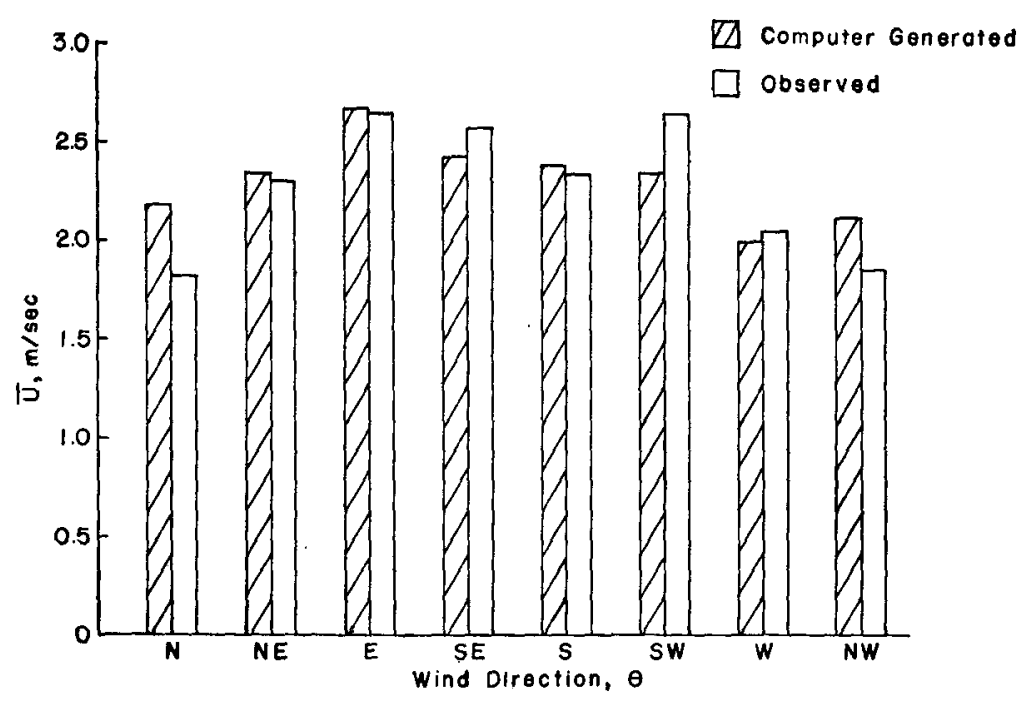

FiG. 5. Comparison of observed and computer-generated wind speeds. 

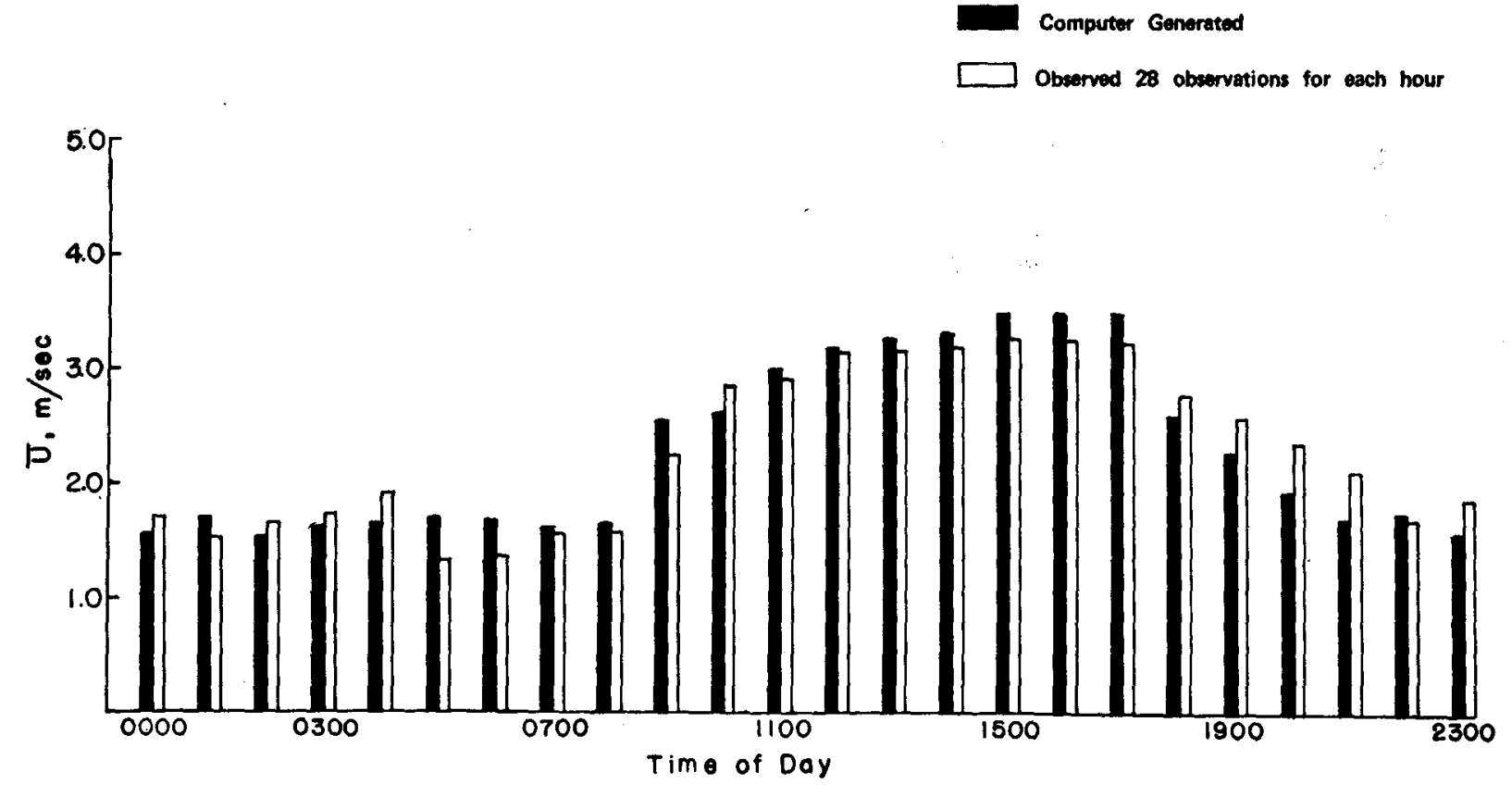

FIG. 6. Comparison of observed and computer-generated diurnal wind speed variations.

Fig. 5 is used to compare observed and computergenerated wind directions for each of the eight principal wind directions. Excellent agreement is obtained between generated and observed values. Fig. 6 shows the comparison between generated and observed diurnal wind speed patterns. Here too, excellent agreement is obtained.

\section{Conclusion}

The results of this study indicate that it is possible to generate wind speed and wind direction stochastically using random number generators of digital computers. When frequency distributions are constructed properly, close agreement between generated and observed values is obtained. When stochastic generation of wind conditions is used in conjunction with appropriate diurnal temperature, stability and mixing depth patterns, it is possible to simulate meteorological conditions encountered during past air pollution episodes. Generating wind conditions stochastically may be preferable to arbitrarily choosing the wind conditions when using an air pollution dispersion model to evaluate the

TABLE 1. Chi-square analysis for observed and generated values of $\theta$ and $\Delta \theta$.

\begin{tabular}{|c|c|c|c|c|}
\hline $\begin{array}{l}\text { Vari- } \\
\text { able }\end{array}$ & $\begin{array}{l}\text { Degrees } \\
\text { of } \\
\text { freedom }\end{array}$ & $x^{2}$ & $\begin{array}{l}\text { Probability } \\
\text { of observing } \\
\text { a larger } \chi^{2}\end{array}$ & Remarks \\
\hline$\theta$ & 7 & 9.65 & 0.22 & $\begin{array}{l}\text { Null hypothesis cannot } \\
\text { be rejected }\end{array}$ \\
\hline$\Delta \theta$ & 7 & 4.31 & 0.74 & $\begin{array}{l}\text { Null hypothesis cannot } \\
\text { be rejected }\end{array}$ \\
\hline
\end{tabular}

effectiveness of different emission control strategies. If an episode is simulated frequently enough to provide a large enough sample size to make statistical inferences, one may obtain a range of meteorological conditions in which to evaluate alternate strategies. The ultimate choice of strategy for air pollution control during emergencies may rest upon effectiveness predicted for different strategies during a majority or certain percentage of episodes simulated. By generating meteorological conditions, at least in part, from tested frequency distributions, one may be assured of obtaining random but realistic meteorological conditions in which to test strategies.

Acknowledgments. Funds for this research were in part provided by Public Health Service Traineeship Grant UI-01026. This financial support is gratefully acknowledged. In addition, the authors wish to thank the Air Pollution Control Office, Environmental Protection Agency for providing useful data concerning past atmospheric stagnation periods.

\section{APPENDIX}

\section{Notation}

$F_{U}$ frequency distribution from which wind speed values are generated

$F_{\delta}$ frequency distribution from which wind speed change values are generated when there is no accompanying change in wind direction

$F_{\Delta} \quad$ frequency distribution from which wind direction change values are generated 
$F_{\theta} \quad$ frequency distribution from which wind direction values are generated

M vector signifying meteorological conditions during a time interval

$T$ composite variable consisting of time of day, atmospheric stability, atmospheric mixing depth and surface temperature

$U$ wind speed

$\theta \quad$ wind direction

$\Delta t$ time interval during which meteorological conditions are assumed to be constant

$\Delta U$ change in wind speed between successive time intervals

$\Delta \theta$ change in wind direction between successive time intervals

\section{REFERENCES}

Bornstein, R. D., 1968: Observations of the urban heat island in New York City. J. A ppl. Meteor., 7, 575-582.
Davis, F. K., and H. Newstein, 1967: Meteorological analysis of the November 1966 and January 1967 air pollution episodes in Philadelphia. Paper presented at Air Pollution Control Association Meeting, Cleveland, Ohio.

DeMarrais, G. A., 1961 : Vertical temperature difference observed over an urban area. Bull. Amer. Meteor. Soc., 42, 548-555.

Duckworth, F. S., and J. S. Sandberg, 1954: The effect of cities upon horizontal and vertical temperature gradients. Bull. Amer. Meteor. Soc., 35, 198-207.

Gifford, F. A., 1952: The breakdown of a low level inversion studied by means of detailed soundings with a modified radiosonde. Bull. Amer. Meteor. Soc., 33, 373-379.

Izumi, Y., 1964: Evolution of temperature and velocity profiles during breakdown of a nocturnal inversion and low level jet. J. Appl. Meteor., 3, 70-82.

Miller, M. E., and L. E. Niemeyer, 1963: Air pollution potential forecasts. J. Air Pollution Control Assoc., 13, 205-210.

Munn, R. E., and T. L. Richards, 1963: Vertical temperature profiles over adjacent land and lake stations. Quart J. Roy. Meteor. Soc., 89, 411-413.

Pooler, F., Jr., 1966: A tracer study of dispersion over a city. J. Air Pollution Control Assoc., 16, 677-681. 